seeded either on plastic or bone slices prior to irradiation to investigate their numbers and function. Osteoblasts (OB) were differentiated from mouse calvariae, irradiated and analysed regarding their differentiation and function using alizarin red stain and $\mathrm{qPCR}$. Hind legs of $\mathrm{hTNF} \alpha$ tg mice were locally irradiated with $0.5 \mathrm{~Gy}$ of X-rays and consecutively analysed using histomorphological methods and $\mu \mathrm{CT}$.

Results Treatment of OC with LD-RT (0.5-2 Gy) lead to decreased differentiation and function, while $\mathrm{OB}$ showed increased mineralization after irradiation with 0.5 Gy. Further, no significant increase of Acp5 and catk at 0.5 Gy was found in OC while OB showed a dose-dependent decrease in RANK$L$ and increase in OPG expression. Also, OPG secretion by $\mathrm{OB}$ was enhanced at $0.5 \mathrm{~Gy}$ and 1 Gy. Histomorphometric analyses showed a decrease of erosive areas and OC numbers in the paws of irradiated animals, a finding that was confirmed in $\mu \mathrm{CT}$ images.

Conclusions We conclude that LD-RT is not only a valuable tool for reduction of inflammation, but also has beneficial effects in restoring a balanced bone homeostasis through increased $\mathrm{OB}$ mineralization and decreased $\mathrm{OC}$ function. In the future, placebo controlled studies are desirable in order to investigate the effects of LD-RT with focus on osteoimmunology in RA in the clinic.

Acknowledgements Supported by the German Federal Ministry of Education and Research (GREWIS, 02NUK017G and GREWIS-alpha, 02NUK050E

Disclosure of interest None declared

\section{P116 HISTONE DEACETYLASE 1: A NOVEL THERAPEUTIC TARGET FOR PATIENTS WITH RHEUMATOID ARTHRITIS}

${ }^{1} \mathrm{~L}$ Göschl, ${ }^{1} \mathrm{~L}$ Müller, ${ }^{1} V$ Saferding, ${ }^{2} \mathrm{~J}$ Bäcklund, ${ }^{3} \mathrm{~S}$ Knapp, ${ }^{4} \mathrm{P}$ Mathias, ${ }^{1} \mathrm{C}$ Scheinecker, ${ }^{5} \mathrm{~W}$ Ellmeier, ${ }^{1} \mathrm{G}$ Steiner, ${ }^{1} \mathrm{M}$ Bonelli*. ${ }^{1}$ Department of Rheumatology, Medical University Vienna, Vienna, Austria; '2Department of Medical Biochemistry and Biophysics, Karolinska Institutet, Stockholm, Sweden; ${ }^{3}$ Research Centre for Molecular Medicine (CeMM), Austrian Academy of Sciences, Vienna, Austria; ${ }^{4}$ Friedrich Miescher Institute for Biomedical Research, Basel, Switzerland; ${ }^{5}$ Division of Immunobiology, Institute of Immunology, Centre for Pathophysiology, Infectiology and Immunology, Medical University of Vienna, Vienna, Austria

\subsection{6/annrheumdis-2018-EWRR2018.131}

Introduction Despite enormous efforts to develop new therapeutic strategies for treatment of rheumatoid arthritis (RA), the large number of non responding patients to currently available drugs underlies the unmet need to identify new therapeutic targets. Certain $\mathrm{CD}^{+} \mathrm{T}$ cells have been shown to be major drivers of inflammation in patients with RA. The expression of their key transcription factors is controlled by histone modifications which includes acetylation of lysine residues mediated by histone deacetylases (HDAC). Indeed, pan HDAC inhibitors have been shown to be a potential therapeutic strategy in the treatment of malignancies. However, major side effects limited the clinical use and underline the need of more specific HDAC inhibitors. Recent data suggest a role of HDAC1 on the migratory capacity and the differentiation of CD $4^{+} \mathrm{T}$ cells in the context of autoimmune encephalitis. ${ }^{1}$

Objectives Our aim was to elucidate the individual role of HDAC1 in a T cell dependent arthritis model, namely the collagen-induced arthritis model (CIA).

Methods Mice with a $\mathrm{T}$ cell specific deletion of HDAC1 (HDAC1 cKO) were generated by using the CD4Cre/LoxP system. At week 8 of age arthritis was induced in wild type
(WT) and HDAC1 cKO mice by immunising with chicken collagen II (CII), emulsified in complete Freund's adjuvant. The animals were scored for clinical signs of arthritis, namely paw swelling and grip strength. Anti-CII antibody levels were determined by ELISA. Various cell subsets, including Th cells, where detected in the blood, the spleen and the draining lymph node by FACS analysis. To test antigen-specific $\mathrm{T}$ cell activation we performed in vitro restimulation of spleen and lymph node cells with collagen II followed by assessment of cytokine production and quantification of the proliferation rate using ${ }^{3} \mathrm{H}$-thymidine incorporation. After 10 weeks mice were sacrificed and paraffin sections of the affected joints were analysed for histomorphologic signs of inflammation, cartilage and bone destruction.

Results Eighty percent of the animals developed serum antiCII anibodies (IgM and $\operatorname{IgG}$ ). No difference in the production of antibody subclasses, especially of pathogenic IgG2c antibodies, were observed between WT and HDAC1 cKO mice. Surprisingly, HDAC1 cKO mice were completely protected and did not develop any clinical signs of arthritis. Accordingly, histological analysis revealed no signs of inflammation, no bone erosion and no appearance of osteoclasts in the joints of HDAC1 cKO mice. In line with our recently published data on the role of HDAC1 on the differentiation and migration of $\mathrm{T}$ cell subsets we suggest that HDAC1 plays a key role in the development of arthritis.

Conclusions Our data show the importance of HDAC1 as a key immune regulator in the pathogenesis of $\mathrm{T}$ cell driven collagen induced arthritis. Therefore it might be considered as an interesting novel therapeutic target in RA.

\section{REFERENCE}

1. Goschl L, et al. A T cell-specific deletion of HDAC1 protects against experimental autoimmune encephalomyelitis. J Autoimmun 2017.

Disclosure of interest None declared

\section{P117 EFFECT OF DIFFERENT JANUS KINASE INHIBITORS ON FIBROBLAST-LIKE SYNOVIOCYTES IN RHEUMATOID ARTHRITIS}

${ }^{1} \mathrm{M}$ Diller*, ${ }^{1} \mathrm{M}$-L Hülser, ${ }^{2} \mathrm{~S}$ Rehart, ${ }^{3,4} \mathrm{M}$ Fleck, ${ }^{1} \mathrm{E}$ Neumann, ${ }^{1} \mathrm{U}$ Müller-Ladner. ${ }^{1}$ Dept. of Rheumatology and Clinical Immunology, Campus Kerckhoff, Justus-Liebig-University Gießen, Gießen; ${ }^{2}$ Dept. of Orthopaedics and Trauma Surgery, Agaplesion Markus Hospital, Frankfurt, ${ }^{3}$ Dept. of Rheumatology and Clinical Immunology, Asklepios Medical Centre Bad Abbach, Bad Abbach; ${ }^{4}$ Dept. of Internal Medicine (I), University Medical Centre Regensburg, Regensburg, Germany

\subsection{6/annrheumdis-2018-EWRR2018.132}

Introduction In 2017, the Janus kinase inhibitors (JAKi) Tofacitinib and Baricitinib were approved for the treatment of Rheumatoid arthritis (RA) in the European Union (EU) by the European Medicines Agency (EMA). Other JAKi like Peficitinib, Filgotinib and Upadacitinib are currently examined in clinical trials. The effect of these substances is mainly based on blocking the IL-6 pathway in immune cells. ${ }^{1}$ However the effect of the different JAKi on activated fibroblast-like synoviocytes from patients with RA (RA-FLS) is not well characterised.

Objectives This study compared the effect of different JAKi on the pro-inflammatory response of activated RA-FLS.

Methods RA-FLS were isolated from synovial tissue of patients with known RA undergoing joint replacement surgery. The 
cells were pretreated with different concentrations of JAKi or vehicle control and then stimulated with IL1- $\beta$ (10 or $20 \mathrm{ng} /$ $\mathrm{ml}$ ) or Oncostatin M (OSM, $100 \mathrm{ng} / \mathrm{ml})$. After the indicated time (17-24 hour), the supernatants were collected and the concentrations of IL- 6 were measured by ELISA. An assay combining the measurement of cell viability, cytotoxicity and apoptosis was performed to exclude effects of JAKi caused by cell toxicity.

Results In a pretest-setting, RA-FLS were pretreated with JAKi for 2 hour with concentrations of $1 \mu \mathrm{M}$ and $10 \mu \mathrm{M}$ and then stimulated with IL1- $\beta(20 \mathrm{ng} / \mathrm{ml})$ for 18 hour. At the concentration of $10 \mu \mathrm{M}$, Peficitinib, Filgotinib and Upadacitinib reduced the IL- 6 release by RA-FLS, whereas Tofacitinib and Baricitinib did not change the IL-6-levels. Tofacitinib and Baricitinib at 1 and $5 \mu \mathrm{M}$ only reduced the cytokine release if the IL-6 pathway was activated selectively with OSM $(n=3)$. In further analyses, RA-FLS were again pretreated with Filgotinib and Peficitinib for 2 hour with clinically relevant concentrations (range between $0.01 \mu \mathrm{M}$ and $5 \mu \mathrm{M}$ ) and then stimulated with IL1- $\beta(10 \mathrm{ng} / \mathrm{ml})$ for 17 hour. At the concentration of $5 \mu \mathrm{M}$, Peficitinib caused a reduction of IL- 6 levels of $66 \%$ compared to control with IL1- $\beta(\mathrm{p}<0.01, \mathrm{n}=5)$. However, a reduction of only $24 \%(\mathrm{p}=0.12, \mathrm{n}=5)$ could be observed at $1 \mu \mathrm{M}$. Filgotinib did not decrease IL-6 levels. Peficitinib did not change the viability, cytotoxicity or apoptosis $(n=2)$, confirming that the observed effects were JAKi dependent.

Conclusions Peficitinib reduced the inflammatory response of RA-FLS after activation with IL1- $\beta$ and appeared to be superior to Tofacitinib and Baricitinib in targeting RA-FLS. The lack of effect of Tofacitinib and Baricitinib on RA-FLS activated by IL1- $\beta$ could explain the treatment failure in some patients.

\section{REFERENCE}

1. Schwartz, et al. Type I/II cytokines, JAKs, and new strategies for treating autoimmune diseases. Nat Rev Rheumatol 2016 January;12(1):25-36.

Disclosure of interest None declared

\section{P118 TRANSFORMING GROWTH FACTOR BETA INDUCED (TGFBI,) A NEW PLAYER IN THE THERAPEUTIC EFFECT OF MESENCHYMAL STEM CELLS}

\footnotetext{
${ }^{1} \mathrm{M}$ Ruiz, ${ }^{2} \mathrm{~K}$ Toupet, ${ }^{1} \mathrm{G}$ Fonteneau, ${ }^{1} \mathrm{M}$ Maumus, ${ }^{3} \mathrm{C}$ Jorgensen, ${ }^{1} \mathrm{D}$ Noel ${ }^{*} .{ }^{1} / \mathrm{nserm}$; ${ }^{2}$ Montpellier University; ${ }^{3}$ Clinical immunology and osteoarticular diseases Therapeutic Unit, CHU Montpellier, Montpellier, France
}

\subsection{6/annrheumdis-2018-EWRR2018.133}

Introduction Osteoarthritis $(\mathrm{OA})$ is the most common form of chronic joint diseases. In recent years, stem cell-based therapies have been investigated as an alternative approach to treat OA. Mesenchymal Stem Cells (MSCs) have demonstrated therapeutic efficacy in the context of pre-clinical studies. More than the capacity of these cells to differentiate into chondrocytes, their effect is primarily associated with a paracrine function.

Objectives Because the Transforming Growth Factor $\beta$ (TGF $\beta$ ) pathway plays a critical role in joint homeostasis, we investigated whether the therapeutic effect of MSCs could be mediated by members of the TGF $\beta$ family. Using a secretome analysis, we identified Transforming Growth Factor $\beta$ Induced (TGF $\beta$ I), a potential candidate for a chondroprotective role of MSCs.
Methods Murine chondrocytes were isolated from 3 days old C57BL/6 mice and cultured for 5 days. OA-like chondrocytes were obtained by incubation with IL1 $\beta$ for 24 hour. Bone marrow-derived MSCs were used to produce a conditioned medium for 24 hour following or not a pre-activation step with TGF 33 for 24 hour. For co-culture experiments, MSCs were seeded on transwells and pre-activated or not with TGF 33 for 24 hour. Supernatants or MSC-containing transwells were added to OA-like chondrocytes (ratio 1 MSC:3 chondrocytes) for 24 hour before cell recovery for RT-qPCR analysis. For silencing experiments, siRNA directed against TGF $\beta$ I were transfected in MSCs and chondrocytes with oligofectamine and lipofectamine, respectively. The CollagenaseInduced OsteoArthritis murine model (CIOA) was induced by collagenase injection in knee joints of $\mathrm{C} 57 \mathrm{BL} / 6$ mice at day 0 and 2. MSCs were transfected twice with siRNA before being injected in knee joints at day $7(250,000$ cells $/ 5 \mu \mathrm{L})$. At day 42 , paws fixed in $4 \%$ formaldehyde and scanned by microCT and confocal laser scanning microscopy before being processed for histology.

Results Addition of conditioned medium from MSCs or MSCs in transwells on OA-like murine chondrocytes allows to reinduce expression of the chondrocyte markers, aggrecan and type IIB collagen. Addition of transwells containing pre-activated MSCs on OA-like chondrocytes not only increased the expression of aggrecan and type IIB collagen but decreased the expression of catabolic markers, MMP13 and ADAMTS5. This effect was associated with an increased expression of TGF $\beta$ I in both chondrocytes and MSCs. We therefore investigated the consequence of TGFßI silencing in either chondrocytes or MSCs. Interestingly, silencing of TGF $\beta I$ in MSCs resulted in a significant reduction of their therapeutic effect. In the CIOA model, down-regulation of TGF $\beta I$ in MSCs resulted in lower therapeutic effect on OA features as visualised by a reduced protective effect on cartilage and sub-chondral bone histomorphometric parameters.

Conclusions Altogether, our results indicated that TGF $\beta I$ secreted by MSCs can regulate cartilage homeostasis by regulating the expression of anabolic and catabolic mediators and that TGF $\beta$ I participates to the protective effect of MSCs in OA.

Disclosure of interest None declared

\section{P119 LOCAL ADMINISTERED ADIPOSE-DERIVED MESENCHYMAL STROMAL CELLS REDUCE EXPERIMENTAL OA-PATHOLOGY VIA INTERLEUKIN-1B- MEDIATED IMMUNOMODULATION OF PRO- INFLAMMATORY PMNS}

${ }^{1} S$ van Dalen, ${ }^{1} \mathrm{M}$ van den Bosch, ${ }^{1} \mathrm{~A}$ Sloetjes, ${ }^{2} \mathrm{~L}$ Casteilla, ${ }^{1} \mathrm{~A}$ Blom, ${ }^{1} \mathrm{P}$ Van Lent ${ }^{*}$ ${ }^{1}$ Experimental Rheumatology, Radboud University Medical Centre, Nijmegen, Netherlands; UMR STROMAlab, University Toulouse, Toulouse, France

\subsection{6/annrheumdis-2018-EWRR2018.134}

Introduction Adipose-derived mesenchymal stromal cells (ASCs) exhibit anti-inflammatory characteristics and reduce development of joint pathology after injection into murine experimental inflammatory osteoarthritis (CiOA) joints. ${ }^{1,2}$ This protection is only achieved when ASCs are applied in early CiOA. This early, but not the late phase of CiOA, is characterised by strongly elevated levels of S100A8/A9 and interleukin-1 beta (IL-1 $\beta),{ }^{3}$ suggesting that the inflammatory environment mediates the protective effect of ASCs. 